\title{
Yōkai als Repräsentanten von Heimat und \\ Japaneseness im Anime Miyori no mori
}

\section{Timo Thelen}

\section{$1 \quad$ Einleitung}

Das Werbeplakat für den Anime-Film Miyori no mori („Miyoris Wald ${ }^{{ }^{1}}$, 2007): Ein unauffälliges junges Mädchen sitzt auf einem Ast inmitten eines grünen und blühenden Waldes, unter ihr fließt ein Bach, im Hintergrund ist das Strohdach eines alten Hauses zu sehen (vgl. Abb. 1). Dieses Bild könnte bis hierhin auch die Werbung für eine Reise in irgendeine ländliche Region in Japan sein.

Ein wichtiger Aspekt wurde jedoch bisher nicht genannt: Um das Mädchen herum tummeln sich unzählige überaus seltsame Kreaturen; die meisten sehen zwar freundlich aus, aber sie erinnern doch an Kobolde, Trolle oder andere Fabelwesen. Wie passen diese mysteriösen Gestalten in das idyllische Landschaftsbild?

Es handelt sich bei diesen Wesen um sogenannte yōkai. Diese japanischen Fabelwesen oder Monster - wenn man yōkai vereinfacht mit einem deutschen Wort beschreiben will - sind seit der Edo-Zeit (1603-1868) ein fester Bestandteil der japanischen Kultur. Sie blicken damit zwar auf eine lange Tradition in der Kunst und Literatur zurück, jedoch werden sie seit Beginn der Moderne (MeijiZeit, 1868-1912) auch gerne in neue Kontexte integriert, und es wird ihnen so eine neue Identität verliehen. So haben sie seit den $1970 e r$ Jahren auch die modernen Populärmedien Anime und Manga erobert.

Der Manga-Zeichner Mizuki Shigeru wird mit seinem Hauptwerk GeGeGe no Kitarō als ein wichtiger Auslöser für den gegenwärtigen yōkai-Boom genannt, der seit den 1990er Jahren durch den Anime-Regisseur Miyazaki Hayao mit seinen Filmen wie „Mein Nachbar Totoro“ (Tonari no Totoro, 1988) und „Chihiros Reise ins Zauberland" (Sen to Chihiro no kamikakushi, 2003) noch größer wurde.

Das Ziel dieses Aufsatzes ist es, anhand eines konkreten Beispiels aufzuzeigen, wie yōkai in der gegenwärtigen Populärkultur dargestellt und verstanden

1 Eine Veröffentlichung außerhalb Japans liegt noch nicht vor. 


\section{Timo Thelen}

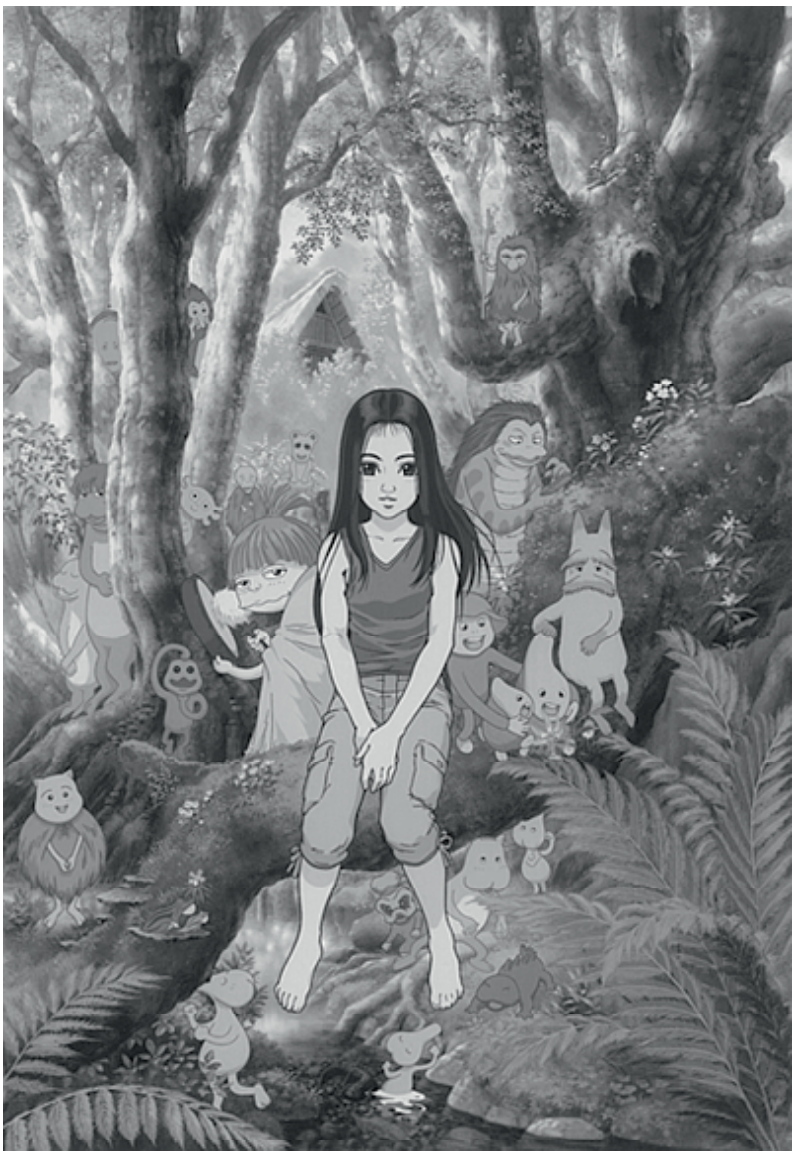

Abbildung 1: Das Werbeplakat zum Film Miyori no mori zeigt die Protagonistin inmitten einer Schar von unheimlichen Wesen. (Quelle: Yuu's World 2009: Internet) 
werden. Es wird untersucht, wie diese ehemals furchterregenden Kreaturen eine positive Umbewertung erfuhren, die sie nun für neue Rollen qualifiziert, die vom niedlichen Kuscheltier bis zum Vermittler einer konservativen Wertehaltung reichen. Dazu wird zunächst als Grundlage kurz beschrieben, wie yōkai vor der Moderne wahrgenommen wurden. Anschließend wird über die neueren Tendenzen in der yōkai-Kultur referiert, bei der auch die japanischen Konzepte von Heimat (furusato) und Japaneseness („Japanizität") eine wichtige Rolle spielen werden. Im zweiten Teil wird der Anime-Film Miyori no mori als Untersuchungsgegenstand in den Fokus gerückt. Nach einer Inhaltsangabe werden die auftretenden yōkai und anderen übernatürlichen Wesen in ihrer Darstellung analysiert und interpretiert.

\section{Die $y \bar{o} k a i$-Kultur}

\subsection{Die unheimliche yōkai-Kultur vor der Moderne}

Zuallererst muss kurz geklärt werden, was überhaupt unter yōkai zu verstehen ist. Komatsu Kazuhiko, der derzeit wohl engagierteste japanische Forscher auf diesem Gebiet, problematisiert, dass der Begriff yōkai sehr vage sei. Er bietet folgende Definition an, die auf den verwendeten Schriftzeichen des Wortes basiert: „[...] ein Phänomen oder eine Existenz, ein Lebewesen, zu dem die Attribute mysteriös, sonderbar, wundersam und unheimlich passen“ (Komatsu 2007: 10). Komatsu vermutet, dass viele yōkai als Erklärungsversuche für Phänomene ( genshō) entstanden sind, die den Menschen seltsam oder unerklärlich erschienen, wie beispielsweise das Geräusch eines umstürzenden Baumes bei Nacht, der am folgenden Tag jedoch nicht entdeckt werden kann (tengudaoshi). Dadurch, dass man solche Erscheinungen als übernatürliche (chōshizenteki) Wesen deklarierte und ihnen einen Namen verlieh, konnten diese Phänomene von einer individuellen zu einer Gruppenerfahrung werden.

Man muss dabei den im alten Japan weitverbreiteten Animismus als Hintergrund sehen, nach dem überall in der Natur Seelen (reikon) existieren. Wenn diese Seelen sich durch Opfer und/oder Gebete besänftigen ließen, sprach man von kami („Gott" oder „Geist“), wenn sie sich keinesfalls vom Menschen kontrollieren ließen, nannte man sie yōkai. Bis diese yōkai aber eine feste visuelle und 
damit wiedererkennbare Gestalt (zōkei) erhielten, dauerte es bis zur Edo-Zeit. Zwar mögen bereits davor vereinzelt grafische Darstellungen existiert haben, aber erst durch die Verbreitung des Druckhandwerks und die lange Friedensperiode in der Edo-Zeit entstand die berühmte japanische yōkai-Kultur (vgl. Komatsu 2006: 12-17).

Als wichtiger Akteur bei dieser Entwicklung wird Toriyama Sekien (17121788) mit seinen vier yōkai-Bildbänden ${ }^{2}$ gesehen. Er war stets bemüht, die yōkai in seinen Zeichnungen in ein überaus dunkles Licht zu rücken und sie zu fürchterlichen Gestalten zu stilisieren. Obwohl in seinem letzten Bildband auch humoristische Ansätze auftauchen, zeigt sich doch in den meisten Fällen beim Vergleich mit älteren Darstellungen - sofern es sich um überlieferte und keine neu erfundenen yōkai handelt - deutlich, dass es Sekiens Anliegen war, seine Kreaturen möglichst unheimlich erscheinen zu lassen. Zahlreiche Holzschnittkünstler wie Kitagawa Utamaro (1753-1806), Katsushika Hokusai (1760-1849), Utagawa Kuniyoshi (1797-1861), Tsukioka Yoshitoshi (1839-1892) und Kawanabe Kyōsai (1831-1889) orientierten sich an seinen Arbeiten und übernahmen die visuellen Merkmale der einzelnen yōkai, wodurch diese zu bekannten und bis heute immer wieder aufgegriffen Archetypen wurden. Dementsprechend verbreitete sich auch die Vorstellung von yōkai als vornehmlich unheimlichen Geschöpfen (Komatsu 2006: 12-17).

\subsection{Yōkai und furusato}

In der Meiji-Zeit bemühte sich Inoue Enryō (1858-1919), yōkai als wissenschaftlich erklärbare Phänomene darzustellen und sie damit „auszurotten“. Nach Enryō mussten die Japaner, um zu zivilisierten und modernen Menschen (kindaijin) werden zu können, begreifen, dass yōkai lediglich Chimären (kakai) seien (Komatsu 2006: 20f). Mit dieser Forderung schafft er gleichzeitig die Vorstellung, dass yōkai im Gegensatz zur Moderne und zum Fortschritt stehen, sie also in die Sphäre der Vergangenheit gehören (Foster 2009b: 114).

2 Gazu hyakki yakō („Bilder der nächtlichen Parade der hundert Dämonen“, 1776), Konjaku gazu zoku hyakki („Bilder der hundert Dämonen von früher und heute“, 1779) und Konjaku hyakki shūi („Ergänzung zu den hundert Dämonen von früher und heute“, 1880) und Hyakki tsurezure bukuro („Nutzloser Beutel mit hundert Gegenständen“, 1784). 
Der Volkskundler Yanagita Kunio (1875-1962) griff diese Tendenz in seiner Sammlung von Volkserzählungen Tōno Monogatari („Die Geschichten von Tōno“, 1910) auf, wo von einem idyllischen Provinzdorf berichtet wird, in dem verschiedene yōkai auftreten. Kunio entwickelt so einen rückwärtsgerichteten Gegenentwurf zur damaligen Realität, die durch die Modernisierung bzw. Anpassung an den Westen geprägt war (Foster 2009b: 139-143). Diese Vorstellung einer Rückkehr zum Leben auf dem Land gehen in Richtung des furusato-Diskurses. Der Begriff furusato lässt sich annähernd mit „Heimat“ übersetzen und kann sowohl auf eine persönliche wie auch eine nationale Ebene angewandt werden. Er wurde durch die Menschen geprägt, die im Zuge der wachsenden Wirtschaft in den 1960er und 7oer Jahren aus ländlichen Gebieten in die Großstädte, vornehmlich Tōkyō, gezogen sind. Angesichts der neuen, oftmals als kalt und abweisend empfundenen urbanen Umgebung begannen sie sich nach ihren Wurzeln zu sehnen. Eine komplexe wertbehaftete Dichotomie zwischen Stadt (Tōkyō) und Land (furusato) entstand, die viele Sphären umfasst, wie beispielsweise die Zeit (Zukunft vs. Vergangenheit), die Nation (Ausland/Internationalisierung vs. ,authentisches Japan“) und das gesellschaftliche Leben (Anonymität/ Egoismus vs. Gemeinschaft) (vgl. Chida 2007: 108-111).

So entstand das Bild eines besseren, aber auch verlorenen Lebensmodells, das gleichzeitig die Unzufriedenheit und Angst gegenüber der Gegenwart ausdrückte (vgl. Robertson 1988: 504). Diese Thematik wurde bald zu einem medialen Gegenstand, der besonders im Volkslied (enka) und in der Werbung aufgegriffen wurde (vgl. Robertson 1988: 496). Ein weiterer kommerzieller Bereich, auf den der furusato-Diskurs einen großen Einfluss ausgeübt hat, ist der Binnentourismus. Projekte wie furusato-zukuri (,furusato erschaffen“) und Themenparks wie furusato-mura (,furusato-Dorf") helfen wirtschaftlich schwachen Regionen zur Erschaffung eines touristischen Potentials. Es ist dadurch ein symbolisches und konsumierbares furusato entstanden (vgl. Creigthon 1997: 249), das auch die jüngeren Generationen anspricht, die selbst keine Vergangenheitserfahrung vom Landleben besitzen. Dieser furusato-Boom in der japanischen Kultur hält bis heute an und ist mittlerweile auch häufig im populären Film und Fernsehen sowie im Manga und Anime zu entdecken. 


\subsection{Furusato und Japaneseness}

Der Diskurs um die Einzigartigkeit der japanischen Kultur und Gesellschaft (Japaneseness) wurde ebenfalls von Yanagita entscheidend geprägt. Er erkannte das besondere, ursprüngliche Japanische in der ländlichen Gemeinschaft mit ihren Umgangsformen, Erzählungen und Bräuchen und erhob diese lokale Kultur zur nationalen Kultur, die bereits zu seinen Lebzeiten durch die sich ausbreitende, westlich geprägte Verstädterung bedroht schien (vgl. Morris-Suzuki 1995: 765ff). Als Reaktion auf die Angst vor einem Identitätsverlust entstand bereits in der ersten Hälfte des 20. Jahrhunderts das literarische Genre nihonron oder nihonjinron („Abhandlungen über die Japaner/über Japan“) (Heise 1990: 7). Dessen Ziel ist die Konstruktion und Behauptung eines homogenen ,japanische[n] Ich[s]“, von dem angenommen wird, dass es „singulär“ und „diskursiv [für den Westen] nicht zugänglich“ sei (Heise 1989: 78f). Die beiden Diskurse furusato und Japaneseness gehen somit auf ähnliche Ursprünge zurück und sind eng miteinander verbunden.

Ivy erkennt, dass alle Diskussionen um nationale kulturelle Identität, Gemeinschaft und Heimat in Japan sich reduzieren lassen auf die Hauptaussage: „[...] ,we Japanese are modern, but we have kept our tradition"“ (Ivy 1995: 26). Bis in die Gegenwart erfreut sich so auch der Japaneseness-Diskurs großer Beliebtheit, denn gerade in der sich globalisierenden Welt, die von modernen Massenmedien und einer stärkeren Migration geprägt ist, wächst in Japan die Angst vor dem Verlust vermeintlicher kultureller Homogenität und gesellschaftlicher Stabilität (Burgess 2004: Internet). Ironischerweise verdankt aber der Anime als eines der bekanntesten Kulturexportgüter Japans - nach Meinung einiger Wissenschaftler - seinen internationalen Erfolg vor allem seiner „Staatenlosigkeit“. Er vertrete für die Ausländer weder Exotik noch Japan, sondern lediglich das moderne Zeitalter (vgl. Berndt 2008: 67). Aber wie im Folgenden analysiert wird, ist diese Annahme nur begrenzt richtig, denn es existieren viele Anime-Filme und Serien, die sich bewusst an ein japanisches Publikum wenden und diesem eine zeitgenössische Interpretation der eigenen Kultur und Tradition vermitteln wollen. 


\subsection{Die freundliche $y \bar{o} k a i$-Kultur in der Gegenwart}

\subsubsection{Mizuki Shigeru}

Der Manga-Zeichner Mizuki Shigeru $\left({ }^{\star} 1922\right)$ wurde in großem Maß von Sekien und Yanagita beeinflusst. Ihre Werke dienten ihm nicht nur als Grundlage für viele Charaktere seiner yōkai-Manga-Serien, er zeichnet sogar neue Versionen von Sekiens Bildbänden und Illustrationen zu den Romanen von Yanagita (vgl. Foster 2009b: 69-73). Das wichtigste Werk von Mizuki ist aber GeGeGe no Kitarō bzw. Hakaba Kitarō („Der Dämonenjunge vom Friedhof“, erste Veröffentlichung 1957 als ausleihbarer Manga), so der ursprüngliche Titel. Der gleichnamige Protagonist ist ein auf dem Friedhof geborener Halb-yōkai (seine verstorbene Mutter war ein Mensch, der Vater ein yōkai) mit menschlicher Gestalt, der zwar unter Menschen aufgewachsen ist, jedoch eindeutig als Verbündeter der yōkai betrachtet werden kann. Das Szenario ist sehr düster gehalten; die menschlichen Figuren werden von vielen verschiedenen yōkai angegriffen und sind ihnen meist unterlegen. Diese erste Version der Serie fand nur wenig Beachtung (vgl. Komatsu 2006: 101). 1968 wurde eine TV-Anime-Adaption ausgestrahlt, gleichzeitig wurde die Serie in einer wöchentlichen Manga-Zeitschrift in überarbeiteter Form veröffentlicht. Der Titel wurde für diese beiden neuen Formate in GeGeGe no Kitarō geändert und der Inhalt für ein breiteres Publikum angepasst.

Die auftretenden Charaktere, egal ob Menschen oder yōkai, können leicht in gute und böse Figuren unterteilt werden. Auch der Protagonist Kitarō hat eine klare Rolle: Er wird zum gutherzigen Verbündeten der Menschen und friedvollen Mediator zwischen Menschen und yōkai. Zusammen mit einer Gruppe von gleichgesinnten yōkai agiert er gegen böse Menschen oder yōkai, wobei viele $y \bar{o}$ kai erst durch das Fehlverhalten von Menschen böse geworden sind; die „Bösen“ sind damit also fast immer die Menschen (vgl. Komatsu 2006: 101-104). Die Anime-Adaption umfasst mittlerweile einige Kinofilme und fünf Staffeln mit über vierhundert Episoden und ist (dank zahlreicher Wiederholungen) seit vierzig Jahren ein ständiger Teil der japanischen Fernsehlandschaft. In den letzten Jahren kamen noch zwei Realverfilmungen ins Kino, und es gab eine TV-AnimeSerie, die sich am düsteren Original-Manga orientiert. Ferner gibt es zahllose 
Merchandising-Produkte wie Spielzeug, Videospiele und sogar Nahrungsmittel, aber auch Ausstellungen und Freizeitparks.

In contemporary Japan, Mizuki's yōkai images are so deeply ingrained in the cultural imagination that you would be hard-pressed to find a child or adult unfamiliar with Kitarō or Mizuki's other paradigmatic creations. (Foster 2009a: 164)

Bei GeGeGe no Kitarō sind auch nostalgische Elemente zu finden. So wohnt Kitarō beispielsweise an einem idyllischen See mitten in einer menschenverlassenen Natur in einem Baumhaus ohne Elektrizität oder sonstige Produkte der modernen Zivilisation. Kitarōs Vater, ein Auge mit winzigem menschlichem Körper, badet immer in einem improvisierten ofuro (traditionelles heißes Bad), einer mit heißem Wasser gefüllten Teeschale. Foster verweist ferner auf Mizukis enge Bindung zu seiner Heimatstadt Sakaiminato in der Präfektur Tottori: „Mizuki lionizes Sakaiminato as a pure furusato or hometown, a nostalgic space metonymic of a time all but forgotten in postwar and postindustrial Japan." (Foster 2009a: 165). Dort gibt es mittlerweile im Rahmen von furusato-zukuri-Maßnahmen viele $y o \bar{k} a i$-Attraktionen wie einen $y \bar{o} k a i$-Schrein und eine Mizuki-ShigeruRoad mit zahlreichen Statuen von den Charakteren aus GeGeGe no Kitarō (vgl. Foster 2009a: 169f).

\subsubsection{Miyazaki Hayao}

Der erste Film, in dem der international bekannte Anime-Regisseur Miyazaki Hayao $\left({ }^{\star} 1941\right)$ yōkai auftreten ließ, ist Tonari no Totoro aus dem Jahre 1988. Dort zieht eine Familie aus der Stadt in ein idyllisches japanisches Dorf, wo die beiden Töchter (zehn und vier Jahre alt) Bekanntschaft mit dem yōkai Totoro machen. Totoro ist ein Mischwesen aus Katze, Eule und Marderhund, das keinen volkstümlichen Ursprung besitzt. Er lebt im Inneren eines alten Baumes im Wald und ist freundlich zu Kindern (Erwachsene können ihn nicht sehen). Miyazaki charakterisiert dieses Wesen in einem Interview mit dem amerikanischen AnimeFanportal Anime-Club wie folgt:

Totoro ist eine Personifikation von Japans Natur [...] und mein Ziel bei diesem Film war es, den Menschen zu zeigen, dass Japan schöner sein sollte. Die Menschen zu warnen, dass sie vorsichtiger sein sollten, oder ihnen zu sagen, dass 
Japan ursprünglich wunderschön war. (Nieder 2004: 65; Übersetzung durch Julia Nieder)

Der erfundene yōkai Totoro ist also als ein Repräsentant der Natur und der Vergangenheit zu verstehen, die Miyazaki als besser einschätzt bzw. so darstellt. Die Geschichte um Totoro spielt daher auch in den 1950er Jahren und damit etwa dreißig Jahre bevor der Film gedreht wurde (vgl. Napier 2001: 473). Dies war genau die Zeit, in der viele Menschen durch die Landflucht ihre Heimat verließen und ihr bald nachtrauerten, wie bereits in Kapitel 2.2 erwähnt wurde. Diese Nostalgie wurde in Tonari no Totoro erneut verarbeitet und macht diesen Anime wohl zu einem der ersten und bekanntesten, der das furusato-Motiv thematisiert. Auch heute noch ist Totoro ein omnipräsentes Geschöpf, vor allem in der Merchandise- und Spielzeugindustrie, und wird für viele japanische Kinder - neben GeGeGe no Kitarō - den ersten und dadurch prägenden Kontakt zu yōkai darstellen. Dabei wird sich nicht das Bild einer unheimlichen Kreatur, sondern das eines naturverbundenen und herzensguten Kuscheltiers festsetzen.

Sen to Chihiro no kamikakushi aus dem Jahre 2001 brachte Miyazaki den internationalen Durchbruch. In diesem Film gelangt das Mädchen Chihiro in eine wundersame Parallelwelt und arbeitet dort in einem traditionellen Badehaus, um ihre in Schweine verwandelten Eltern zu retten. Diese Parallelwelt kann als eine magische Version der japanischen Vergangenheit bezeichnet werden - obwohl sie auch einige westliche und chinesische Elemente enthält (vgl. Napier 2006: 290). Chihiro dient dabei als Identifikationsfigur für den Zuschauer, dem diese traditionelle Sphäre mit volkstümlichem Charakter (matsuri) und yōkai ebenso fremd sein mag. Miyazaki erklärt im Begleitheft zum Film: „Die [heutigen] Kinder sind von Hightech umgeben und verlieren inmitten der oberflächlichen Produkte ihre Wurzeln. Wir müssen ihnen vermitteln, wie reich unsere Tradition ist." (Komatsu 2006: 115).

Gegen dieses Vergessen der „eigenen Wurzeln“ betreibt Miyazaki laut Napier eine "activist nostalgia“ (Napier 2006: 293), die zur Revitalisierung der japanischen Tradition aufruft und die in direkter Verbindung zum furusato-Diskurs steht: „Miyazaki's vision of Japanese traditional Culture is clearly linked to the notion of furusato" (Napier 2006: 293). Figal hebt bei dieser Nostalgie im be- 
sonderen Maße das „Wundersame“ hervor. Er sagt, Sen to Chihiro no kamika$k u s h i$ habe die Tendenz „[to] fetishize the fantastic, reifying fragments of fushigi [„wundersam"] into whole cultural manifestations that could then be identified as authentically Japanese.“ (Figal 1999: 220f). Diese „wundersamen“ und gleichzeitig „rein japanischen“ Elemente stellen im Wesentlichen die yōkai dar, die wie bei Mizuki nicht nur als Bestandteil, sondern auch als Übermittler der Vergangenheit angesehen werden können.

\section{Miyori no mori}

Der Anime Miyori no mori basiert auf dem gleichnamigen Manga von Oda Hideji $\left({ }^{*} 1962\right)$, der 2003 zunächst als Serie in einer Zeitschrift erschien, danach aber im gleichen Jahr auch als einbändige Ausgabe. Der hier betrachtete AnimeSpielfilm wurde unter der Regie von Yamamoto Nizō $\left({ }^{*} 1953\right)$ für den Fernsehsender Fujiterebi gedreht und am 25.08.2007 zur Primetime ausgestrahlt sowie Ende desselben Jahres auch als DVD veröffentlicht. Auffällig sind das für einen TV-Anime verhältnismäßig hohe Budget von 210 Million Yen (etwa 1,5 Millionen Euro), die dreijährige Produktionsdauer und die Beteiligung von vielen Mitgliedern des berühmten Anime-Studio Ghibli, dem auch Miyazaki angehört. Die Fernsehausstrahlung hatte jedoch trotz einer großen Werbeoffensive nur eine mittelmäßige Zuschauerquote von $15,8 \%$ in der Kansai- und $17,2 \%$ in der Tōkai-Region (vgl. Cinema Topics 2010: Internet).

\subsection{Inhalt}

Nach der Trennung der Eltern (die Mutter ist ausgezogen) bringt der Vater die etwa zwölfjährige Miyori zu ihren Großeltern in ein ländliches Dorf. Zunächst ist sie mit ihrer neuen Umgebung sehr unzufrieden und will zurück nach Tōkyō. In der Schule wird sie zwar größtenteils freundlich aufgenommen, aber sie hasst den Rummel um ihre Person als Mensch aus der Stadt. Als ihr eines Nachts einige friedliche yōkai begegnen, läuft sie verängstigt weg. In einem Gespräch mit ihrer Großmutter, die von den Dorfbewohnern als gute Hexe (majō) bezeichnet wird, erfährt sie, dass auch sie von den yōkai weiß und dass Miyori ihre Nachfolge als 
„Schutzgöttin des Waldes“ (mori no mamorigami) antreten soll. Daraufhin öffnet sich Miyori den yōkai, erinnert sich an eine erste Begegnung mit ihnen in ihrer frühen Kindheit und schließt mit ihnen Freundschaft.

Eines Tages gelangt sie im Wald an eine Quelle, die von dem Geist einer verstorbenen Frau besetzt wird, der im Konflikt mit den yōkai steht. Diese Frau hatte einst an der Quelle Selbstmord begangen, weil sie von Männern betrogen wurde. Von diesem Geist erfährt Miyori, dass der Wald und das Dorf bald durch einen Dammbau überflutet werden sollen. Die yōkai bestätigen ihr dies. Miyori erzählt einem Mitschüler von der Quelle, der anschließend dorthin geht. Miyori eilt ihm hinterher, kann ihn vor dem wütenden Geist retten und ihn schließlich erlösen.

Um den Wald zu retten, wollen Miyori und ihre Schulfreunde in der Umgebung Steinadler (inuwashi) suchen, die als stark bedrohte Art gelten und deren Existenz den Dammbau aufgrund von Artenschutzvorschriften verhindern würde. Miyori beauftragt auch die yōkai, einen Steinadler zu suchen, aber sie muss bald erkennen, dass keine mehr in diesem Wald leben. Miyoris Mutter erscheint plötzlich im Dorf und will ihre Tochter überreden, mit ihr zusammen zurück nach Tōkyō zu gehen. Nach vielen Streitigkeiten einigen sie sich darauf, dass Miyori nur wiederkommt, wenn ihre Eltern wieder zusammen leben. Die Mutter verspricht ihr daraufhin, noch einmal mit dem Vater zu reden.

Einige Fremde tauchen im Dorf auf, die anscheinend mit dem Dammprojekt in Verbindung stehen. Als man ihnen von den Steinadlern erzählt, suchen sie auch nach ihnen. Ein yōkai verwandelt sich in einen Steinadler und erscheint vor den Männern, die daraufhin mit Gewehren auf ihn schießen. Es stellt sich heraus, dass es in Wahrheit Jäger sind, die die bedrohten Tiere des Waldes töten sollen, damit der Dammbau ohne Hindernisse stattfinden kann. Miyori ruft alle yōkai zusammen und will mit ihrer Hilfe die Jäger verscheuchen, die in der folgenden Nacht mit Verstärkung wiederkehren. Bei ihrer Rückkehr werden sie von den yōkai und Miyori als ihrer Anführerin erschreckt und vertrieben. Die verängstigten Jäger werden am Waldrand von der Polizei aufgegriffen. Nach ihrem Erfolg beschließen die yōkai, zeitweise als Steinadler aufzutreten, um den Wald in Zukunft zu schützen. Weil Miyori jedoch voraussieht, dass die Men- 
schen in einigen Jahren oder Jahrzehnten wieder versuchen werden, den Wald zu zerstören, will sie für immer dort bleiben und ihn verteidigen.

\subsection{Yōkai oder seirei?}

Zunächst stellt sich die Frage, ob die auftretenden übernatürlichen Geschöpfe überhaupt noch unter die Kategorie yōkai fallen. Im Film bezeichnen sie sich selbst als seirei (39:00) („Elementargeist“, „, höherer Geist“), Miyori nennt sie mori no sei („Waldgeister“, 39:00), und sie zeigen sich ihr gegenüber in ihrer „,harmlosen“ Gestalt. Beim Treffen mit den Jägern nehmen sie hingegen ihre „unheimliche Form“ (kowaku hengen suru, 1:32:00) an, um diese zu erschrecken. Die Jäger sprechen deswegen von bakemono (1:36:00), was etwa bedeutungsgleich mit yōkai ist. Man mag lange darüber streiten können, welcher Begriff insgesamt für diese Gestalten der passendste ist. Wenn man sich an Komatsus Definition orientiert, die in Kapitel 2.1 erläutert wurde, nach der übernatürliche Wesen in kami und yōkai durch das Unterscheidungskriterium, ob sie sich durch Gebete und/ oder Opfer besänftigen lassen, eingeteilt werden können, würde es sich um $y \bar{o}$ kai handeln. Es ergibt sich aber das Problem, dass der historische Kontext dieser Einteilung nach Komatsu heute nicht mehr gegeben ist, denn es wird von eher unheimlichen und böse gesinnten yōkai ausgegangen, wie sie in der Edo-Zeit und davor gesehen wurden. Die wundersamen Waldbewohner sind stattdessen in der neueren Tendenz, die durch Mizuki und Miyazaki geprägt wurde, einzuordnen, nach der yōkai meist als freundliche und niedliche Geschöpfe dargestellt werden.

Noch ein wichtiges Argument spricht für die Bezeichnung yōkai: Viele der Waldbewohner sind an bekannte yōkai-Darstellungen angelehnt. Kanoko (auf Abb. 1 rechts neben Miyoris Kopf) ist deutlich als kappa, eine Art Flusskobold, zu erkennen. Er wacht nach der Erlösung des Geistes über die Quelle und attackiert am Ende die Jäger aus dem Wasser, er versucht, sie hineinzuziehen; dieses Verhalten kennt man aus Überlieferungen vom kappa (vgl. Foster 1998: 6). Auch gibt es ein zashiki warashi mit Namen Washirashi (auf Abb. 1 links neben Miyoris Arm), einen Hausgeist, der in regionalen Erzählungen auftaucht. Ein Waldbewohner, Bokuriko (auf Abb. 1 rechts neben Miyoris Arm), besitzt die 
Gabe des Gestaltenwandelns (bakeru), die im Volksglauben viele yōkai wie zum Beispiel der tanuki (Marderhund) und der kitsune (Fuchs) beherrschen (vgl. Harada 1976: 1). Somit gibt es mehrere Argumente dafür, bei den übernatürlichen Wesen in Miyori no mori von yōkai zu sprechen.

\subsection{Onryō und kannon-Buddha}

Neben den yōkai treten noch zwei andere übernatürliche Wesen auf. Eines ist der Geist, der die Quelle besetzt. Leider wird er während des Films nur als „Frau“ (onna, 39:00) bezeichnet, allerdings wäre yūrei oder shiryō (beides bezeichnet den Geist eines Verstorbenen), bzw. onryō (nicht ruhender Rachegeist) eine passendere Beschreibung. Diese „Frau“ entspricht dem klassischen Bild des weiblichen Rachegeistes in der japanischen Kultur. Sie trägt ein langes weißes (Toten-) Kleid, ist barfuß und kann schweben. Sie hat einst an dieser Stelle Selbstmord begangen, weil sie von Männern ausgenutzt und betrogen wurde, und aufgrund ihres grausamen Todes und ihres großen Zorns (urami) konnte ihr Geist keine Ruhe finden. Die Menschen scheinen von diesem Geist zu wissen, Miyoris Mitschüler erzählen von ihm und an der Stelle, wo die Frau gestorben ist, hängen Papierzacken (shide) - ein Shintō-Brauch, um Orte, an denen Geister oder Götter leben, zu kennzeichnen. Als Miyori ihr alleine begegnet, besitzt sie eine menschenähnliche, nicht unheimliche Gestalt, durch das weiße Kleid aber auch ein typisches Merkmal für einen Geist. Erst als ein Mitschüler von Miyori an die Quelle gelangt, verändert sich ihr Gesicht, sie bekommt eine schauderhafte Fratze mit spitzen Zähne und Hörnern. Diese Darstellung erinnert sehr an die standardisierte Maske eines weiblichen Geistes (hannya) im Nō-Theater. Miyori kann sie besiegen und erlösen, indem sie ihr ihren tragischen Fehler vor Augen führt. Nicht die Männer waren schuld an ihrem Tod, sondern ihre eigene Schwäche. Mit dieser Erkenntnis kann ihr Geist in die Totenwelt einkehren; sie bedankt sich bei Miyori und verschwindet.

Das zweite übernatürliche Wesen, das nicht unter die Kategorie yōkai fällt, ist der „Geist (oder die Göttin) des einzelnen Kirschbaumes“ (ippon sakura no sei, 1:23:00). Die yōkai nennen sie ihre „Ratgeberin“" (washi-ra no hosasha, 1:23:00) und sie war es auch, die Miyori zur "Schutzgöttin des Waldes“ erkoren hat. Ihr 
Aussehen ähnelt der Ikonographie eines kannon-Buddhas . Ihre Kleidung, vor allem der Schmuck auf dem Kopf, an den Armen und die Halskette sowie die Frisur erinnern an diesen Buddha-Typus, wenn auch einige kleinere Unterschiede zu erkennen sind, z.B. dass der kannon-Buddha zumeist mit einer Lotusblüte in der Hand dargestellt wird, was hier nicht der Fall ist. Zudem trägt sie Blüten am Kopfschmuck, was ihre Verbundenheit zur Natur symbolisiert und womit man sie ebenfalls im Kontext der mutterähnlichen Figur des kannon-Buddhas sehen kann oder auch im Rahmen der westlichen Mythologie als eine Art „Mutter Natur“. Obwohl sie eines der mächtigsten Wesen des Waldes zu sein scheint, ist sie beim Kampf gegen die Jäger nicht beteiligt. Sie heilt lediglich den verwundeten yōkai, der in Gestalt eines Steinadlers angeschossen wurde. Im Gegensatz zu den yōkai scheint sie, wie auch der onryō, von den Menschen wahrgenommen und verehrt zu werden, denn um den Stamm ihres Kirschbaumes ist ebenfalls ein Seil mit shide gebunden. Sie fällt, obwohl sie als sei bezeichnet wird, am ehesten unter die Kategorie göttliches Wesen (kami oder Buddha).

\subsection{Furusato-Motive}

In Miyori no mori lassen sich die beiden gegensätzlichen Welten Stadt (Tōkyō) und Land (Dorf) eindeutig erkennen. Auch wenn der Schauplatz des Anime lediglich das Dorf ist, wird durch die Erinnerungen von Miyori sowie durch die Figuren ihrer Eltern der zweite Ort, Tōkyō, mit seinen charakteristischen Merkmalen indirekt konstruiert. Das Leben in Tōkyō bzw. die Menschen dort werden dabei äußerst negativ dargestellt. Miyoris Eltern können als stereotype Vertreter der Stadtmenschen gesehen werden, die keinen Vor- oder Nachnamen besitzen und immer nur Mutter und Vater genannt werden. Sie streiten sich in Miyoris Erinnerungen ständig, meist weil ihr Vater neben seiner Arbeit keine Zeit für die Familie findet und weil die eigensüchtige Mutter es bedauert, für den Kinderwunsch ihre eigene Karriere aufgegeben zu haben. Die Mutter fühlt sich im ländlichen Dorf sehr unwohl, sie mag das Essen nicht und will bei jedem Besuch schnellstmöglich wieder wegfahren. Sie raucht, ist westlich gekleidet, hat gefärbte Haare, wirkt arrogant und oberflächlich. Sie verlässt ihre Familie plötzlich und flieht damit auch vor ihrer (traditionellen) Rolle als Hausfrau und Mut- 
ter. Miyoris Vater mag zwar das Dorf, in dem er früher gelebt hat, aber sagt bei jedem Besuch, dass er wegen seiner Arbeit nicht lange bleiben kann. Er trägt immer Hemd und Brille und erscheint wie ein stereotyper salaryman ${ }^{3}$, der nur für seine Arbeit lebt und sich deshalb auch nicht um die Erziehung von Miyori kümmert. Die anderen Menschen in Tōkyō erscheinen ebenfalls als tendenziell schlecht und egoistisch. In einer Erinnerungssequenz wird Miyori in ihrer damaligen Schule gemobbt (ijime), und sie sagt später zu den Menschen im Dorf, dass sie keine Freunde in Tōkyō hatte. Die Jäger als stereotype Menschen, die die Natur nicht schätzen, kommen ebenfalls aus Tōkyō.

Die Dorfbewohner hingegen werden als äußerst positive Menschen präsentiert. Sie nehmen die fremde Miyori überaus freundlich in ihre Gemeinschaft auf (ein Junge mobbt sie zwar zu Beginn, aber nach dem Zwischenfall mit dem Geist an der Quelle werden auch sie Freunde). Diese Gemeinschaft umfasst Menschen jeden Alters, denn in der Schule scheinen alle - trotz verschiedenen Alters - zusammen Sportunterricht zu haben und verbringen auch die Freizeit zusammen. Die Großeltern von Miyori, die wie ihre Eltern nicht mit Namen vorgestellt werden, sind ein harmonisches Paar, das ein gewisses Ansehen im Dorf besitzt; die Großmutter hat den Ruf, eine gute Hexe zu sein. Eine ähnlich positive Gemeinschaft bilden auch die yōkai im Wald, die meist in großen Gruppen auftreten und die untereinander keine Konflikte haben (abgesehen vom Streit mit dem Geist an der Quelle). Das in 2.2 beschriebene komplexe Gegensatzpaar Tōkyō furusato kann besonders im Punkt des gesellschaftlichen Lebens wiedererkannt werden (Anonymität/Egoismus vs. Gemeinschaft).

Die Idylle des Lebens auf dem Land wird ebenfalls deutlich betont. Lange Sequenzen des Anime zeigen die unberührte Natur des Waldes, der auch auf dem Titelbild ein großer Raum zugesprochen wird. Das Dorf mit seinen alten Strohdachhäusern aus Holz und den fruchtbaren Reisfeldern wird oft als Panorama eingeblendet. Als Miyori dort ankommt, wirft sie ihr Handy weg, weil es keinen Empfang hat. Die einzigen modernen Produkte, die in dem Dorf auftauchen, sind die Autos von Miyoris Eltern und den Jägern, die also der antagonistischen

3 Festangestellter in einer mittelständischen bis großen Firmen, lange Zeit gleichbedeutend mit einem erfolgreichen und der gesellschaftlichen Norm entsprechenden Lebensweg. 
Sphäre Tōkyō angehören. Miyoris Großeltern wohnen in einem traditionellen Haus, das z.B. nicht über westliche Betten verfügt (bis der Großvater gegen Mitte des Films für Miyori eins baut), dafür aber über ein heißes Bad (ofuro) und eine offene Feuerstelle (irori). Auf dem Speicher lagern viele okkulte Gegenstände, nicht nur aus Japan, sondern auch aus Nordasien und Europa, über die der freundliche Hausgeist wacht.

Ein Leben in Harmonie mit der Natur und dem Spirituellen wird aufgezeigt, das die Menschen aus Tōkyō (Miyoris Mutter und die Jäger) in keiner Weise zu schätzen wissen. Diese traditionell-japanische dörfliche Welt steht im Kontrast zum modernen, technisierten und internationalisierten Tōkyō, das die ländlichen („authentisch japanischen“) Wurzeln scheinbar verloren hat. Die in $\mathbf{2 . 2}$ beschriebenen Gegensatzpaare Vergangenheit vs. Zukunft und Japan vs. Ausland/Internationalisierung als erweiterte Sphären der furusato-Tōkyō-Dichotomie sind hier klar wiederzuerkennen.

\subsection{Die Interpretation der yōkai}

Die auftretenden yōkai sind nicht unheimlich, denn sie stehen kaum mehr in Verbindung zur yōkai-Kultur der Edo-Zeit. Stattdessen sind sie im Kontext der modernen Umdeutung, wie sie bei Mizuki und Miyazaki zu finden ist, anzusiedeln.

Sie sind zwar ein versteckter, nur für wenige Bewohner wahrnehmbarer Teil der dörflichen Gemeinschaft, aber ein entscheidender, weil durch ihre Hilfe der Wald und das Dorf vor dem Dammbau gerettet werden können. Sie repräsentieren den Wald und die Natur, die durch den Fortschritt der menschlichen Zivilisation gefährdet sind. Miyori übernimmt dabei die wichtige Rolle des Bindeglieds zwischen den Menschen und den yōkai bzw. der Natur. Genau genommen ist sie eine Art Überläuferin aus der modernen Welt (Tōkyō), die sich im Laufe der Geschichte immer bewusster für ein Leben auf dem Lande (furusato) entscheidet. Nur durch ihre menschliche Hilfe können der Wald und das Dorf gerettet werden, weil sie (neben ihrer Großmutter) die einzige Person ist, die noch einen Zugang zu der mächtigen Sphäre des Spirituellen in der Natur besitzt. Beim Kampf gegen die Jäger am Ende des Films trägt sie keine menschlich- 
zivilisierte Kleidung mehr, sondern nur noch eine Art Fell und schlägt auf eine mystische Trommel. Ihr Aussehen erinnert dabei stark an San aus Mononokehime (dt. Titel: „Prinzessin Mononoke“, 1997), einem Anime von Miyazaki, der im Mittelalter spielt und der den Kampf zwischen einer expandierenden Stadt und einem dadurch bedrohten Wald schildert. San ist in diesem Film die Anführerin der Waldbewohner (hauptsächlich Tiere, aber auch einige yōkai), ein Mädchen, das unter Wölfen aufgewachsen ist und sich in der Wildnis wohler fühlt als in der menschlichen Zivilisation. Auch in diesem Film siegt, allerdings nach einer wesentlich blutigeren Schlacht als bei Miyori no mori, am Ende die Natur.

Die Göttin des Kirschbaumes hat Miyori auserkoren, die „Schutzgöttin des Waldes“ zu werden, obwohl - oder gerade weil - sie ein Mensch ist. Die Botschaft, die hier suggeriert wird, ist leicht verständlich: Der Mensch muss die Natur schützen und retten, die Natur kann dies trotz ihrer übernatürlichen Bewohner nicht alleine. Aber dieses Verhältnis ist noch komplexer, denn die Natur ist untrennbar mit dem ländlichen Dorf, dem Ort der japanischen Tradition und Vergangenheit, verbunden, der mit ihr zusammen zu verschwinden droht. Deswegen geht es nicht nur um eine bewusste Entscheidung für den Schutz und die Erhaltung der Natur, sondern auch um die Bewahrung der eigenen Traditionen wie der Religiosität (Glaube an yōkai, onryō und kannon-Buddha) und der sozialen Wertvorstellungen (Gemeinschaft und Familie als wichtige Einheiten und Bezugspunkte für die Menschen), die in der ländlichen Vergangenheit verwurzelt sind. Es geht also - mit anderen Worten - um die japanische Identität bzw. Japaneseness, die vor dem Fortschritt und damit vor dem Verschwinden geschützt werden soll. Damit schließt sich nicht nur der Bogen zu den Werken von Mizuki und Miyazaki, sondern bis zu Enryō und Yanagita, und es zeigt sich die noch immer währende Aktualität des Diskurses über den Verlust einer japanischen Identität angesichts der Internationalisierung.

\section{Fazit}

Yōkai entwickelten sich in der menschlichen Vorstellung von unerklärlichen Naturphänomenen zu übernatürlichen Wesen, die in der Edo-Zeit eine feste und wiederkennbare Gestalt erhielten. Vor allem Sekien ist als wichtiger Akteur zu 
nennen, der maßgeblich zum Entstehen der unheimlichen yōkai-Kultur beigetragen hat und der bis in die Gegenwart existente Archetypen geschaffen hat. Mit dem Beginn der Moderne forderte der Intellektuelle Enryō die Abschaffung dieser Chimären, die seiner Meinung nach den Fortschritt der Nation behinderten. Der Volkskundler Yanagita machte unter diesem Einfluss yōkai zum Bestandteil einer urjapanischen Vergangenheit auf dem Lande, indem er sie in seinen gesammelten Erzählungen als Bestandteil einer traditionellen Dorfgemeinschaft charakterisierte. Nach dem Zweiten Weltkrieg kam es zu einem neuen yōkaiBoom, für den im Wesentlichen Mizuki verantwortlich gewesen ist, der seinen yōkai-Helden Kitarō zu einem friedvollen Verbündeten der Menschen machte. Miyazakis erfundener yōkai Totoro hat ebenfalls alles Unheimliche aus der yōkai-Kultur der Edo-Zeit abgelegt und lebt, ähnlich wie Kitarō, in einer idyllischen Landschaft jenseits der modernen Großstädte. Der furusato-Diskurs muss hierbei als wichtiger Hintergrund betrachtet werden. Seit den 1960er/7oer Jahren entwickelte sich im Denken vieler Japaner eine komplexe und stark wertebehaftete Dichotomie zwischen der ländlichen Heimat (furusato) und der als kalt empfundenen Großstadt (meist Tōkyō). Diese Nostalgie wird bis heute sowohl im Binnentourismus als auch in den Medien thematisiert. Auch Sen to Chihiro no kamikakushi von Miyazaki beschäftigt sich mit dem Heimats- und Identitätsverlust der modernen Japaner und begreift die yōkai als ein magisches Element der traditionellen Vergangenheit.

Der Anime Miyori no mori steht im Kontext dieser neuen Tendenzen in der gegenwärtigen yōkai-Kultur. In diesem Film lernt die junge Miyori das ländliche Japan als einen viel harmonischeren und schöneren Ort kennen als das anonyme und feindliche Tōkyō. Diese lebenswertere Umwelt ist allerdings bedroht. Zur Rettung des Dorfes und des Waldes, die als Sinnbild für die Tradition, Naturverbundenheit und Gemeinschaft gesehen werden können, erhält sie die wichtige Unterstützung der yōkai. Diese yōkai sind gute Waldgeister, zu denen die meisten Menschen keinen Zugang mehr besitzen, die aber ohne die Hilfe der Menschen nicht weiter existieren können. Der Anime zeigt beispielhaft den Konflikt zwischen den beiden Welten Tōkyō und furusato auf. Während Tōkyō für eine internationale, entwurzelte und egoistische Zukunft steht, symbolisiert 
furusato das „echte Japan“ mit seiner ländlichen und traditionsreichen Vergangenheit, die nicht erst heute, sondern bereits seit Beginn der Modernisierung Japans Ende des 19. Jahrhunderts gefährdet ist.

Yōkai besitzen zwar seit der Edo-Zeit eine bekannte und konstante visuelle Form, ihr Inneres ist aber je nach Zeitgeist neu definierbar, da sie letztlich vom Menschen geschaffene Fantasiegeschöpfe sind. Damit ist zu erklären, dass die ehemals unheimlichen Kreaturen aus der Vormoderne nun als freundliche Vermittler der furusato-Ideale und Japaneseness-Vorstellung fungieren können. Diese Tendenz ist zwar nicht vollkommen neu, wie man an den Werken von Yanagita erkennt, dennoch erlebt sie besonders seit den 1990er Jahren ein beeindruckendes Revival. Dabei sollte auch der gesellschaftliche Kontext berücksichtigt werden, denn nicht nur Japan, sondern die ganze Welt befindet sich in einem stark globalisierten Zustand, in dem die Nationalstaaten zwar immer noch als starre Grenzen wahrgenommen werden, sich aber tatsächlich immer mehr einander anpassen und damit auch die Identität und die Vergangenheit einzelner Völker und Kulturen ineinander übergehen lassen. Deswegen wird gerade im heutigen Japan das Ideal einer „rein-japanischen“ Heimat konstruiert, in das alles zu fallen scheint, was als traditionell oder nicht-ausländisch gilt. So eben auch die yōkai.

\section{Filmographie}

Tonari no Totoro, „Mein Nachbar Totoro“. Regie: Miyazaki Hayao. Japan: Studio Ghibli, 1988.

Mononoke hime, „Prinzessin Mononoke“. Regie: Miyazaki Hayao. Japan: Studio Ghibli, 1997.

Sen to Chihiro no kamikakushi, „Chihiros Reise ins Zauberland”. Regie: Miyazaki Hayao. Japan: Studio Ghibli, 2001.

Miyori no mori, „Miyoris Wald“. Regie: Yamamoto Nizō. Japan: Nippon Animation, 2007.

\section{Literatur}

BERNDT, Jaqueline (2008): „Zeichentrick-Japanisch: Japanimation und Anime“. In: Berndt, Jaqueline; Richter, Steffi (Hg.): Japan-Lesebuch IV: J-culture. Tübingen: Konkursbuchverlag. S. 48-70. 
Creighton, Millie (1997): „Consuming Rural Japan: The marketing of Tradition and Nostalgia in the Japanese Travel Industry”. In: Ethnology 36 no. 3. S. 239-254.

FIgAL, Gerald (1999): Civilisation and Monsters - Spirits of Modernity in Meiji Japan. Durham: Duke University Press.

Foster, Michael Dylan (2009a): „Haunted Travelogue: Hometowns, Ghost Towns, and Memories". In: Mechademia, Volume 4, 2009. S. 164-181.

Foster, Michael Dylan (2009b): Pandemonium and parade - Japanese monsters and the culture of yōkai. Berkley/Los Angeles: University of California Press.

Foster, Michael Dylan (1998): „The metamorphosis of the kappa: Transformation of folklore to folklorism in Japan”. In: Asian Folklore Studies, Vol. 57, No. 1. S. 1-24.

Harada, Violet H. (1976): „The badger in Japanese folklore”. In: Asian Folklore Studies, Vol. 35 , No. 1. S. 1-6.

Heise, Jens (1989): „Nihonron - Materialien zur Kulturhermeneutik”. In: Menzel, Ulrich (Hg.): Im Schatten des Siegers: JAPAN Kultur und Gesellschaft. Frankfurt a. M.: Suhrkamp. S. 76-97.

HeISE, Jens (1990): Die kühle Seele - Selbstinterpretationen der japanischen Kultur. Frankfurt a. M.: Fischer.

Ivy, Marilyn (1995): Discourses of the Vanishing - Modernity, Phantasm, Japan. Chicago: University of Chicago Press.

Komatsu, Kazuhiko (2006): Yōkai bunka nyūmon. („Einführung in die yōkai-Kultur”). Tōkyō: Serika shobō.

Morris-Suzuki, Tessa (1995): „The Invention and Reinvention of Japanese Culture”. In: The Journal of Asian Studies 54, No. 3. S. 759-780.

NAPIER, Susan J. (2006): „Matter Out of Place: Carnival, Containment and Cultural Recovery in Miyazaki's Spirited Away”. In: Journal of Japanese Studies 32.2. S. 287-310.

Nieder, Julia (2004): Die Filme von Hayao Miyazaki. Marburg: Schüren.

Robertson, Jennifer (1988): „Furusato Japan: The Culture and Politics of Nostalgia”. In: International Journal of Politics, Culture, and Society, Vol. 1, No. 4. S. 494-518.

\section{Internetquellen}

Burgess, Chris (2004): „Maintaining Identities - Discourses of Homogeneity in a Rapidly Globalizing Japan”, http://www.japanesestudies.org.uk/articles/Burgess.html [Stand: $5 \cdot 3 \cdot 2011$ ].

CHIDA, Tomoko (2007): „Furusato ron saikō“ („Nachdenken über den furusato-Diskurs“). In: Ochanomizu Joshidaigaku: Ochanomizu chiri, 37 (Ochanomizu-Frauenuniversität: Ochanomizu Geografie 37): 108-114. http://teapot.lib.ocha.ac.jp/ocha/bitstream/ 10083/12304/1/003710.pdf [Stand: 24.6.2010].

Cinema Topics: „ミヨリの森DVD“(Miyori no mori); http://www.cinematopics.com/cinema/ present/premora.php?number $=1000$ [Stand: 7.3.2011].

YUU's WORLD (2009): „ミヨリの森“(Miyori no mori); http://blogs.yahoo.co.jp/setu_choko/ 466433.html [Stand: 4.5.2011]. 